\title{
Actitudes hacia los niños adoptables por parte de familias monoparentales y nucleares de estratos 4, 5 y 6 en la ciudad de Bogotá
}

\author{
Estefanía Abello Forero \\ Estudiante de Psicología \\ Universidad de San Buenaventura, Bogotá \\ Colombia \\ Correo electrónico: laur.a39@hotmail.com

\begin{abstract}
Jhoana Carolina Bello Pulido
Estudiante de Psicología

Universidad de San Buenaventura, Bogotá

Colombia
\end{abstract} \\ Correo electrónico: jhoanacbello@hotmail.com \\ Angie Güiza Suarez \\ Estudiante de Psicología \\ Universidad de San Buenaventura, Bogotá \\ Colombia \\ Correo electrónico: An_yita@hotmail.es
}

\footnotetext{
Ana María Ortiz Escobar

Estudiante de Psicología

Universidad de San Buenaventura, Bogotá

Colombia

Correo electrónico: anamari0120@gmail.com
}

\author{
Fernando Riveros Munévar \\ Mg. en Psicología \\ Universidad de San Buenaventura, Bogotá \\ Colombia \\ Correo electrónico: efriveros45@hotmail.com
}

\author{
Luis Anderssen Vera Maldonado \\ Mg. en Educación \\ Universidad de San Buenaventura, Bogotá \\ Colombia \\ Correo electrónico: Ivera@usbbog.edu.co
}

Arleth Patricia Bernal Vásquez
Magíster en Neuropsicología clíica
Universidad de San Buenaventura, Bogotá
Colombia
Correo electrónico: abernal@usbbog.edu.co

Liliana Reyes Gómez

Magíster en comunicación

Universidad de San Buenaventura, Bogotá

Colombia

Correo electrónico: Ireyes@usbbog.edu.co

Recibido: 05/09/2017

Evaluado: 07/11/2017

Aceptado: 19/04/2018

\section{Resumen}

La presente investigación tiene como objetivo describir las actitudes hacia los niños adoptables por parte de familias nucleares y monoparentales de estratos 4, 5 y 6 en la ciudad de Bogotá. Cuenta con un enfoque cuantitativo y un tipo de estudio descriptivo-comparativo. La muestra total fue de 107 familias, 57 familias nucleares y 50 monoparentales, bajo un muestreo no probabilístico, teniendo en cuenta las edades de los padres entre los 30 a 45 años y que no estuviesen implicadas dentro de un proceso de adopción. Se aplicó el Test de Actitudes hacia el Niño Adoptable (TANA), compuesto por 20 ítems. Los resultados indicaron que las familias nucleares tuvieron mejores actitudes a nivel cognitivo y conductual y, adicionalmente, mejores actitudes hacia la salud, temperamento y género del niño adoptable en comparación con las familias monoparentales, no siendo significativas las diferencias en cuanto a edad y etnia.

Palabras clave

Actitudes, adopción, Familia nuclear, familia monoparental.

3 Para citar este artículo: Abello, E., Bello, J., Güiza, A., Ortiz, A.M., Riveros, F., Vera, L.A., Bernal, A.P., \& Reyes, L. (2018). Actitudes hacia los niños adoptables por parte de familias monoparentales y nucleares de estratos 4, 5 y 6 en la ciudad de Bogotá. Informes Psicológicos, 18(2), pp. 45-62 http://dx.doi.org/10.18566/infpsic.v18n2a03 


\section{Attitudes towards adoptable children by single- parent and nuclear families of strata 4, 5 and 6 in the city of Bogotá}

\section{Abstract}

The objective of this research is to describe the attitudes toward adoptable children by nuclear and single-parent families of strata 4, 5 and 6 in the city of Bogotá. A quantitative approach descriptive-comparative study type was carried out. The total sample was of 107 families, 57 nuclear families and 50 single-parent families. A nonprobabilistic sampling was applied, taking into account the ages of the parents, who were between 30 and 45 years old and were not involved in an adoption process. The Test of Attitudes toward Adoptable Children (TANA) composed of 20 items was applied. The results indicated that the nuclear families had better attitudes at the cognitive and behavioral level and, in addition, better attitudes toward the health, temperament and gender of the adoptable child compared to single-parent families, not there being significant differences in age or ethnicity.

Keywords

\section{Atitudes em relação ás crianças adotáveis por parte de famílias monoparentais e nucleares de estratos sociais 4, 5, 6 na cidade de Bogotá}

Resumo

A presente pesquisa tem como objetivo descrever as atitudes em relação ás crianças adotáveis por parte de famílias nucleares e monoparentais de estratos 4, 5 e 6 na cidade de Bogotá. Conta com um enfoque quantitativo e um tipo de estudo descritivo-comparativo. A amostra total foi de 107 famílias, 57 famílias nucleares e 50 monoparentais, sob uma amostra não probabilística, levando em consideração as idades dos pais entre os 30 e os 45 anos e que não estivessem implicadas em um processo de adoção. Foi aplicado 0 teste de atitudes em relação à criança adotável (TANA), composto por 20 itens. 0s resultados indicaram que as famílias nucleares tiveram melhores atitudes no nível cognitivo e condutual e, além do mais, melhores atitudes com a saúde, o temperamento e 0 gênero da criança adotável em comparação com as famílias monoparentais, não sendo significativas as diferencias com relação a idade e a etnia.

Palavras chave Atitudes, adoção, família nuclear, família monoparental. 


\section{ntroducción}

En el mundo y en Colombia a lo largo del tiempo ha coexistido la niñez en desamparo y son muchos los menores que esperan en instituciones de adopción para ser acogidos en el seno de una familia; sin embargo, entran en juego diversos factores, como las características del niño adoptable: salud, edad, género, temperamento y raza, que, a su vez, podrían llegar a ser un motivo para su difícil adopción (López \& Garrido, 2009). De acuerdo a lo anterior, el hecho de que el deseo de muchos niños de ser adoptados no se cumpla, puede deberse a las diferentes actitudes que tenga cada familia en relación a las particularidades que presenten los mismos, estando en la base de esta problemática los padres que no cumplen su rol dentro de las funciones de crianza y educación de sus hijos, tomando la decisión de abandonarlos y dejarlos bajo el cuidado del Estado, en lugar de protegerlos y brindarles la atención que requieren (Palacios, 2009).

Por su parte, la adopción se considera como una de las alternativas excepcionales para restituir los derechos de niños en condiciones de vulnerabilidad (López \& Garrido, 2009). De igual forma, desde un punto de vista legal, Pérez (2016) afirma que la adopción es "el estado jurídico mediante el cual se confiere al adoptado la situación de hijo del o de los adoptantes, y a estos, los deberes y derechos inherentes a la relación paterno-filial. Es el vínculo filial creado por el derecho" (p. 131).

En adición, la Corte Constitucional colombiana (2011) reconoce que el concepto de familia no solo abarca la constituida por un matrimonio de hombre y mujer (nuclear), sino que existen diferentes modalidades de conformarla, entre ellas las familias monoparentales, es decir, aquella conformada por una madre o padre y sus hijos. La familia nuclear reúne la mayor proporción de familias en Colombia, evidenciando una vigorosa disminución en tanto incrementan los hogares con un solo progenitor (familia monoparental), siendo la viudez, el madre-solterísmo y la separación conyugal aspectos que explican este hecho (Alonso, 1999).

Por otra parte, si bien dentro de un proceso de adopción es fundamental el papel de la familia adoptante que realiza la solicitud de adopción, es indispensable la presencia de un niño adoptable a espera de llegar a ser un hijo en el seno de una respectiva familia. Basándose en lo anterior, el niño adoptable es todo menor de 18 años que puede ser considerado para su adopción al ser declarado en abandono (Palacios, 2009).

Cabe resaltar que un aspecto fundamental y común a todo tipo de adopción es el papel que desempeña la familia, la cual, según sostiene UNICEF (2006), es el primer baluarte de protección del menor. Adicionalmente, Meneses, Arenas y Pino (2005) afirman que es el primer espacio socializador del ser humano, donde se dan los primeros aprendizajes, se desarrolla el afecto y la satisfacción de necesidades sexuales, sociales, emocionales y económicas, así como se crean, trasmiten y reciben normas, valores, símbolos, ideologías e identidades (Alonso, 1999).

En lo que se refiere a la familia monoparental, la obligación del progenitor de 
desempeñar un papel dual en la paternidad al cuidar a sus hijos, lleva consigo grandes gastos económicos, con lo que aparece la necesidad de la ayuda de las familias extendidas. Reflejo de estas condiciones es el hecho de que la modalidad de familia nuclear pesa mucho más en los sectores socioeconómicos medio y alto, ubicándose en el bajo el triple de la proporción de jefes de hogar sin cónyuge, con sus hijos (Rosser \& Moya, 2001).

Por su parte, los niños adoptables en muchas ocasiones presentan deficiencias físicas, bien sea debido a que provienen de orfanatos con condiciones socioeconómicas que involucren inseguridad, o dada su procedencia de familias con bajos recursos que susciten grandes problemas que pueden afectar negativamente su salud y crecimiento (Bustamante, 2013).

De igual forma, Palacios (2009) reconoce que en los niños adoptables existen algunas particularidades, ya que "hay niños y niñas, bebés y mayores, solos y con hermanos, sin y con problemas importantes (de salud, psicológicos, educativos...)" (p. 20).

En vista de estas características con las que provienen los menores adoptables, el papel que vienen teniendo los padres adoptivos en la vida de los niños es cumplir sus funciones de supervivencia proporcionándoles un hogar, y teniendo una vinculación afectiva puesto que, además de los problemas de salud, estos niños pueden manifestar conductas agresivas, antisociales, de oposición etc., rendimiento académico bajo y problemas de atención e hiperactividad (Bustamante, 2013).
Según estudios de la UNICEF (2016) que tenían como fin evaluar la incidencia global de la adopción internacional, se evidenció que entre 170.000 y 180.000 niños se vieron implicados en procesos de adopción internacional entre 1980 y 1989. En ese periodo, la adopción aumentó en un 62\% proviniendo el 90\% de los niños de 10 países. En consecuencia, las estadísticas actuales señalan una notable reducción del número de niños adoptados en los últimos años, pasando de una proporción de 10.277 niños desde el 2012 a 778 a la fecha del 2015, siendo muchos de los niños que habitan en los hogares de protección, aquellos cuyas características se consideran especiales, debido a que pueden hacer parte de un grupo numeroso de hermanos, son mayores de 8 años o presentan alguna discapacidad o problemas de salud permanente. Teniendo en cuenta esto, se podría afirmar que la difícil adopción de los menores estaría más asociada a sus características que a la incertidumbre que surge de los padres adoptivos de no sentirse capaces de suplir la totalidad de las necesidades de su nuevo hijo. En este sentido, conviene subrayar que la edad es uno de los aspectos que más caracteriza a estos menores, pues a la fecha de 2015, 4.850 niños con necesidades especiales estaban en espera de adopción, siendo el 69\% (3.337) de ellos de edades entre los 13 y 18 años y el 31\% restante menores de 12 años (Instituto Colombiano de Bienestar Familiar, ICBF, 2015).

Teniendo como base las consideraciones anteriores, el ICBF (2015) reportó la existencia de 368 solicitudes de adopción de familias colombianas en espera de niños y niñas sanos con edades entre 0 y 6 años de edad y 2.281 solicitudes de familias extranjeras en lista de espera, 
de las cuales 123 aceptarían niños con algún estado leve o recuperable de salud, demostrando que estas familias son más receptivas a recibir a los niños, inclusive con problemas de salud. Por su parte, Bogotá es la ciudad en la que mayor número de niños hay en espera y que, a su vez, ejerce más participación en la adopción en un 16\%, siendo los menores sanos la mayor parte de los niños adoptados (aproximadamente el 67\%), por lo cual, el temor al cuidado y manejo de niños con características especiales, hace menor la posibilidad para su adopción.

En correspondencia a las cifras de adopción, Miranda (1999) afirmó que los colombianos son rigurosos al adoptar a un niño, ya que en la mayoría de los casos desean adoptar niñas considerando que la crianza de ellas es más sencilla que la de los niños. En la misma medida, Cordero, Yánez y Falconi (2013) señalan que los padres tienden a esperar de sus hijos patrones físicos, intereses o actitudes similares a los de ellos, como si fuera un hijo biológico.

De acuerdo a lo anterior, se podría considerar que el hecho de que muchos de los menores no sean adoptados por familias monoparentales esté más asociado con la valoración que hacen éstas con respecto a la idoneidad de la adopción, más que por las características propias del niño. Bajo estas circunstancias se podría traer a colación lo que mencionan Cordero et al. (2013) expresando que podría haber incertidumbre del padre o madre de no poder sentirse capaz de suplir la totalidad de las necesidades básicas y afectivas para su nuevo hijo.

En la misma medida, Aguirre, Hurtado, Márquez y Lombana (2007) afirman que la adopción de un niño(a) se facilita de acuerdo a las características y circunstancias que rodean su historia de vida. No obstante, ante el hecho de que muchos menores sean difíciles de adoptar y varios de ellos presenten características especiales no se podría suponer que su difícil adopción se deba en su totalidad a sus particularidades; en este sentido, la adopción podría depender también de la organización de la familia adoptante, pues no todas son iguales. Así, en el caso de familias monoparentales, el solo hecho de que quienes adopten sean personas viudas, separadas o personas solteras lleva consigo un conjunto de factores que las hace diferentes a otros estilos de familias como el aumento de inversiones en los hijos en tiempo y dinero, incremento de factores estresantes (acumulación de obligaciones, valoración negativa por parte de la sociedad, carencia de recursos financieros, etc.) y el asumir un papel dual que, en ocasiones, no se consigue (Rosser \& Moya, 2001). Además, se encuentra que las actitudes hacia estos niños en condición de adoptabilidad son también relevantes para el proceso.

Para el propósito de comprensión, las actitudes se definen como reacciones positivas, negativas o mixtas, propias de cada individuo hacia diversas personas o grupos sociales particulares, objetos e ideas (Kassin, Fein \& Rose, 2010; Riveros, Bohórquez, López \& Sepúlveda, 2015). Así mismo, son adquiridas por medio de aprendizajes básicos a través de procesos inmediatos y automáticos que tienden a influir en el comportamiento, siendo susceptibles al cambio, es decir, una vez incorporada la actitud, no significa que ésta sea definitiva. 
Con referencia a lo expuesto anteriormente, para desarrollar una actitud de forma coherente y consecuente, es necesario tener en cuenta los tres factores que la componen: el afectivo, el cognitivo y el conductual o tendencia a la acción. En primer lugar, respecto al componente cognitivo, está formado por un conjunto de creencias, valores, conocimientos, opiniones y expectativas que tienen un orden y relación entre sí. Seguidamente, el componente afectivo, se trata de una predisposición a favor o en contra, donde se abarcan sentimientos y afectos que una persona experimenta hacia un objeto de actitud. Por último, el componente conductual o tendencia a la acción, son las disposiciones conductuales que se manifiestan a manera individual en pro o en contra de un objeto de actitud (Riveros et al., 2015; Robbins, 2004).

En este sentido, respecto a los niños en condición de adoptabilidad, las actitudes hacia estos se definirían como las reacciones positivas, negativas o mixtas, propias de cada individuo hacía aquellos menores de 18 años que pueden ser considerados para su adopción al ser previamente declarados en desamparo.

En la misma medida, dado que las actitudes son propias de cada individuo, son las familias quienes, en este caso, presentan las actitudes hacia el niño adoptable, pues son éstas las que toman la decisión de adoptar y, al hacerlo, como afirma $\mathrm{Pa}$ lacios (2009), cada individuo se hace cargo de todas las características, la historia y el pasado del menor, al integrarlos en la vida familiar y en la relación con éste.

En cuanto a los estudios realizados respecto a las actitudes hacia el niño adoptable, Dave Thomas Foundation (2007) y
Forward Scout (2015) revelaron que la mayor parte de los norteamericanos y australianos manifestaban una actitud favorable frente a estos niños, al mismo tiempo que adoptaban opiniones extremadamente positivas hacia la adopción. Sin embargo, se evidencian diferencias en los hallazgos de estos estudios en cuanto a los conocimientos que se tiene acerca de los niños adoptables, pues mientras que en los hallazgos de Dave Thomas Foundation (2007), los norteamericanos parecían tener un conocimiento erróneo al creer que estos niños están bajo protección por delincuencia, los hallazgos de Forward Scout (2015) revelan que los australianos tienen un conocimiento más acertado considerando que estos menores son dignos de atención y están allí por abandono, maltrato o negligencia de los padres o cuidadores y no por culpa propia.

Es importante señalar que los norteamericanos manifestaban preocupaciones al percibir los problemas de salud, emocionales y comportamentales de los niños y sentirse incapaces de pagar por su cuidado y educación. Ahora bien, menos de la mitad de los australianos encuestados por Forward Scout (2015) consideraban que estos menores tienden a ser difíciles, siendo este punto coherente con la creencia de que estos menores estaban bajo cuidado del Estado no por delincuencia, aspecto que, por el contrario, no adoptaban los norteamericanos al creer que los niños eran delincuentes. Con todo, los australianos no pretendían adoptar y, de los pocos que pensaban en hacerlo (17\%), la mayoría no procedió (83\%).

Posteriormente, y en consonancia con los hallazgos anteriores, Dave Tomas Fundation (2013) pone de manifiesto que los norteamericanos aún revelan intenciones 
altruistas (querer darles amor a los niños necesitados) como algunas de las razones para adoptar, evidenciándose aún la existencia de creencias erróneas como pensar que los niños en condición de adoptabilidad tienden a tener problemas de vinculación, comportamiento y autocontrol. Por otro lado, los hallazgos manifestaron una percepción de mayor probabilidad de adopción de niños menores de 3 años, teniendo una percepción negativa de adoptabilidad de un niño mayor de 12 años, al mismo tiempo que se evidencia una preferencia por la adopción de niñas y una percepción de la raza como un aspecto secundario al considerar la opción de adoptar.

En lo que se refiere a familias residentes en Bogotá, estudios como los de Aguirre et al. (2007), Castañeda (2004) y Riveros (2008), cuyos participantes eran tanto solteros como casados con hijos, padres adoptivos (ya adoptaron) y padres pre adoptantes, hallaron actitudes tanto positivas como neutrales con tendencia positiva hacia los niños adoptables, con una predisposición a aceptar sus características, adoptando la mayoría de los participantes una percepción de solidaridad y altruismo hacia estos menores, lo cual concuerda parcialmente con los hallazgos previamente mencionados cuyos participantes eran extranjeros.

Así mismo, Aguirre et al. (2007) hallaron que las familias colombianas, al igual que las extranjeras, pese a la alta percepción de favorabilidad, no toman la decisión de adoptar, evidenciándose un nivel medio de disposición a la adopción (54.5\%). En consecuencia, esto es posible dado el pensamiento únicamente emocional hacia la adopción, considerándola como una forma de caridad, más que uno racional de involucrarse a sí mismo en ella, o, por el contrario, un pensamiento dirigido a la satisfacción de las necesidades propias; destacando razones por las que no se opta por adoptar tanto el temor al origen genético, la historia de vida del menor con sus efectos en la salud y conducta, además de la competencia de los adoptantes para afrontar las dificultades que genera esta situación, como la demanda económica (más si el niño presenta condiciones particulares en su desarrollo).

En contraposición, Castañeda (2004), al medir las actitudes, obtuvo en promedio una actitud positiva en todos los componentes; indicando que hay mayor prevalencia de pensamientos, ideas y sentimientos positivos hacia características tales como el proceso de adopción, adaptación del niño al entorno, edad y antecedentes; así mismo se evidencia que existe más disposición a adoptar sin dar relevancia a la raza, antecedentes y procesos de selección del niño. En este sentido, Riveros (2008), a pesar de hallar una actitud positiva en la escala total de actitudes, las puntuaciones de las subescalas indican, en promedio, una actitud positiva en relación a la edad evolutiva y personalidad del niño, así como una actitud neutral en cuanto al género, salud y grupo étnico.

Respecto a las diferencias en cuanto a las actitudes de familias nucleares y monoparentales, Aguirre et al. (2007) encontraron que los solteros con hijos estuvieron más dispuestos a adoptar (75\%) que los casados con hijos (50\%), afirmando, además, que la mayoría de quienes estaban dispuestos a adoptar deseaban menores de 5 años, argumentando la facilidad de adaptación del menor a la familia y para guiar su formación, al tiempo que se mostraron indiferentes respecto a la etnia. 
Adicionalmente, Riveros (2008) evidenció diferencias en las actitudes de personas casadas, solteras, viudas y divorciadas en cuanto a la etnia del menor, sobre todo en las actitudes de familias monoparentales y nucleares.

En síntesis, según la literatura, se puede afirmar que las actitudes acerca de las características del niño adoptable, en su componente cognitivo, indican conocimientos en muchas ocasiones erróneos; en su componente afectivo, se aprecia una favorabilidad, y conductualmente no existe una clara disposición de las familias a adoptar, sin embargo, las familias de solteros con hijos poseen una mayor disposición a hacerlo. Además, se evidencian diferencias en las actitudes dependiendo de la familia en relación a la etnia del menor.

Teniendo en cuenta las estadísticas y hallazgos en la literatura, se refleja en las familias adoptantes prejuicios como actitudes negativas, caracterizados por una percepción sesgada de la familia hacia los niños en condición de abandono al basarse únicamente en el hecho de que estos pertenezcan a un grupo social particular (Myers, 2000), siendo el prejuicio sutil el que más se podría presentar en estas familias, pues si bien aparentan en muchas ocasiones percibir favorablemente a estos menores, no adoptarían a un menor con determinadas características manifestando de manera indirecta su prejuicio al no demostrar abiertamente sus emociones o afectos positivos o negativos (Frías, Monterde i Bort \& Peris, 2009).

Ahora bien, la no adopción de estos niños también puede venir asociada a esquemas que se tengan hacia tales menores siendo los esquemas, en esencia, marcos mentales que permiten organizar grandes cantidades de información de una forma eficiente que, a su vez, pueden tener efectos en el pensamiento social al ser moldeados por la cultura. En la misma medida, tanto los esquemas como los prejuicios podrían estar implicados en la determinación de las conductas prosociales como el hecho de adoptar, pues estas conductas representan cualquier acto que beneficia a otras personas (Baron, 2005). Finalmente, cabe resaltar que los hallazgos de esta investigación pueden servir como aporte a futuras investigaciones.

Con base en los fundamentos establecidos anteriormente, se genera como problema de investigación la siguiente pregunta: ¿Cuáles son las actitudes hacia los niños adoptables por parte de familias monoparentales y nucleares de estratos 4, 5 y 6 en la ciudad de Bogotá?, cuyo propósito general es describir las actitudes hacia estos niños por parte de familias monoparentales y nucleares de estratos altos en la ciudad de Bogotá y, de forma más específica, comparar dichas actitudes según el tipo de familia.

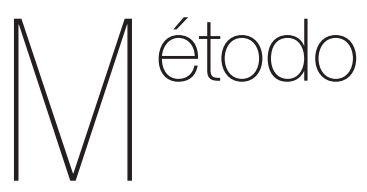

\section{Diseño}

Se trata de una investigación cuantitativa de tipo descriptivo por comparación de grupos, ya que se describen las actitudes de familias monoparentales y nucleares y las diferencias entre éstas respecto a esta variable (Hernández, Fernández, \& Baptista, 2014). 


\section{Participantes}

Se seleccionan 107 familias bajo un muestreo no probabilístico por cuotas, de las cuales 57 son familias nucleares o tradicionales y 50 son familias monoparentales. Para su participación se tienen en cuenta los siguientes criterios: que tengan edades entre los 30 y 45 años, que su estrato económico sea alto $(4,5$ y 6$)$ y que no sean solicitantes de adopción.

\section{Instrumento}

Para medir las actitudes se empleó el Test Evaluativo de Actitudes hacia el Niño Adoptable (TANA), diseñado en el marco de la investigación, el cual contó con una confiabilidad total del .80 por medio del Coeficiente Alfa de Cronbach. Su finalidad fue medir las actitudes que la familia tiene respecto a lo que siente, piensa y actúa frente a los niños adoptables. Está compuesto por 20 afirmaciones cuya escala es tipo Likert. De las afirmaciones, 6 hacen referencia al agrado o desagrado (lo que se siente); 7 a los conocimientos, opiniones y creencias (lo que se piensa) y 7 a las intenciones o tendencias a reaccionar (lo que se hace) respecto a la edad, salud, etnia, temperamento y género del niño adoptable.

\section{Procedimiento}

Para recolectar los datos para la respectiva investigación, se llevaron a cabo 3 fases. Primero se ubicaron a las familias y se estableció contacto con ellas con el objetivo de darles una descripción del estudio y obtener su autorización como participantes de éste. En segundo lugar, se procedió a aplicar el instrumento de actitudes hacia la adopción en un lugar cómodo y adecuado para responder a las afirmaciones sin interrupciones y, finalmente, una vez recolectados los datos, se recopilaron en una base de datos y se procedió a su respectivo análisis estadístico con el programa SPSS.

\section{Consideraciones éticas}

En función al cumplimiento de la Resolución 8430, en su artículo 11, se sustenta que la investigación se clasifica con riesgo mínimo, ya que no se realizan prácticas que atenten contra la libertad e integridad física y psíquica de los participantes y para efectos del código de ética del psicólogo (Ministerio de Protección Social, Ley 1090 de 2006), se garantiza la confidencialidad de la información de los participantes regida por el artículo 23, donde se resalta que la información obtenida por el profesional será guardada en secreto, sin difundirla fuera del estricto marco para el que fue recabada. Así mismo, atendiendo al artículo 28, se garantiza que la información adquirida no será utilizada para beneficio propio o de tercero, ni para el prejuicio de la persona interesada.

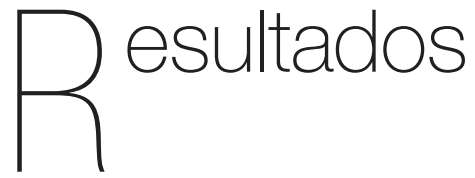

Teniendo en cuenta las puntuaciones arrojadas por el instrumento, se puede afirmar que en la mayoría de las 57 familias nucleares (49.1\%) y de las 50 familias 
monoparentales (52\%), predomina una actitud negativa hacia la edad del niño adoptable, siendo mayor la proporción de familias monoparentales con esta actitud, lo que lleva a afirmar que en ambos tipos de familias se aprecia una tendencia a reaccionar de forma negativa frente al hecho de que el niño sea menor de 5 años o mayor de los 8 (Tabla 1).

Tabla 1

Frecuencias de actitud según familia

\begin{tabular}{cccccccccccc}
\hline \multirow{2}{*}{ Tipo de familia } & \multicolumn{3}{c}{ Edad } & \multicolumn{2}{c}{ Etnia } & \multicolumn{2}{c}{ Salud } & \multicolumn{2}{c}{ Temperamento } & \multicolumn{2}{c}{ Género } \\
\cline { 2 - 12 } & & F & $\%$ & F & $\%$ & F & $\%$ & F & $\%$ & F & $\%$ \\
\hline \multirow{3}{*}{ Familia Nuclear } & Negativa & 28 & 49.1 & 6 & 10.5 & 16 & 28.1 & 1 & 1.8 & 7 & 12.3 \\
& Neutra & 23 & 40.4 & 13 & 22.8 & 30 & 52.6 & 12 & 21.1 & 20 & 35.1 \\
& Positiva & 6 & 10.5 & 38 & 66.7 & 11 & 19.3 & 44 & 77.2 & 30 & 52.6 \\
\hline \multirow{3}{*}{ Familia Monoparental } & Negativa & 26 & 52 & 11 & 22 & 25 & 50 & 7 & 14 & 5 & 10 \\
& Neutra & 19 & 38 & 6 & 12 & 23 & 46 & 12 & 24 & 33 & 66 \\
& Positiva & 5 & 10 & 33 & 66 & 2 & 4 & 31 & 62 & 12 & 24 \\
\hline
\end{tabular}

Nota: F: Frecuencia; P: Porcentaje

Al observar los datos de la Tabla 1, respecto a la etnia, más de la mitad de familias nucleares $(66.7 \%)$ y monoparentales (66\%) tomaron una actitud positiva. De acuerdo a estos datos, se podría suponer que, en ambos tipos de familias, la mayor parte tiende a reaccionar de forma favorable frente al hecho de que el menor sea afrocolombiano, indígena, raizal o gitano, o sea de alguna etnia distinta a la de la familia.

Los resultados indican que, respecto a la salud, en las familias nucleares predominó una actitud neutra (52.6\%) en comparación de las monoparentales en las cuales se presentó, en mayor medida, una actitud negativa (50\%). En este sentido, se puede afirmar que las familias monoparentales tienden a reaccionar con mayor negatividad al hecho de que el menor adoptable tenga o no discapacidades o enfermedades físicas o mentales, siendo la salud del niño adoptable una característica irrelevante para las familias nucleares.

En relación al temperamento, según los hallazgos, en ambos tipos de familias predominó una actitud positiva, siendo el $77.2 \%$ de las familias nucleares y el $62 \%$ de las familias monoparentales quienes presentaron esta actitud. Así, es posible afirmar que, en esencia, los dos tipos de familias tienden a reaccionar con favorabilidad ante el hecho de que el niño sea extrovertido o introvertido.

Finalmente, en cuanto al género, la mayor parte de las familias nucleares presentaron una actitud positiva (52.6\%), al tiempo que predominó en las familias monoparentales una actitud neutra (66\%). Por otra parte, cabe resaltar que el género fue la única característica en la cual el porcentaje de personas con actitud negativa fue mayor en las familias nucleares (12.3\%). De acuerdo a estos datos, 
se podría afirmar que la mayor parte de las familias nucleares tienden a reaccionar con favorabilidad frente al género del niño, al tiempo que para las familias monoparentales no es relevante esta característica. La Tabla 2 refiere la actitud por cada componente.

Tabla 2

Frecuencias de actitud según componente

\begin{tabular}{|c|c|c|c|c|c|c|c|}
\hline \multirow{2}{*}{\multicolumn{2}{|c|}{ Tipo de familia }} & \multicolumn{2}{|c|}{ Afectivo } & \multicolumn{2}{|c|}{ Cognitivo } & \multicolumn{2}{|c|}{ Conductual } \\
\hline & & $\mathrm{F}$ & $\%$ & $\mathrm{~F}$ & $\%$ & $\mathrm{~F}$ & $\%$ \\
\hline \multirow{4}{*}{ Familia Nuclear } & Negativa & 6 & 10.5 & 5 & 8.8 & 7 & 12.3 \\
\hline & Neutra & 45 & 78.9 & 44 & 77.2 & 36 & 63.2 \\
\hline & Positiva & 6 & 10.5 & 8 & 14 & 14 & 24.6 \\
\hline & Total & 57 & 100 & 57 & 100 & 57 & 100 \\
\hline \multirow{4}{*}{ Familia Monoparental } & Negativa & 7 & 14 & 11 & 22 & 7 & 14 \\
\hline & Neutra & 42 & 84 & 39 & 78 & 40 & 80 \\
\hline & Positiva & 1 & 2 & 0 & 0 & 3 & 6 \\
\hline & Total & 50 & 100 & 50 & 100 & 50 & 100 \\
\hline
\end{tabular}

En lo que se refiere a cada uno de los componentes de las actitudes (Tabla 2), los hallazgos indican que, en la mayoría de las familias, tanto nucleares como monoparentales, predominó una actitud neutra a nivel afectivo $(78.9 \%$ de las familias nucleares y el $84 \%$ de las monoparentales), cognitivo $(77.2 \%$ de las nucleares y el $78 \%$ de las monoparentales) y conductual (63.2\% de las nucleares y el $80 \%$ de las monoparentales). Acorde a estos datos, se puede afirmar, por un lado, que ambos tipos de familias no tienden a presentar sentimientos $y$ afectos o tener creencias, conocimiento, opiniones y expectativas a favor ni en contra frente a cada una de las características del niño adoptable (edad, género, salud, etnia y temperamento) y, por otro, no están ni dispuestas a adoptar a estos menores ni tampoco a dejarlos en abandono.
Mencionadas las actitudes hacia cada característica del niño adoptable, y para conocer si dichas diferencias son significativas, se procede a determinar si la distribución de las puntuaciones es normal. Con este propósito se realizó el análisis mediante la prueba de Kolmogorov-Smirnov dado que el tamaño de la muestra es mayor a 50 familias, no encontrándose una distribución normal en las variables de actitud ( $p<.05)$, así mismo, al segmentar la muestra por tipo de familia. En vista de la no normalidad de la distribución, se procede entonces a emplear la prueba no paramétrica $U$ de Mann Whitney para determinar si hay o no diferencias en los grupos de comparación de familias monoparentales y nucleares.

Posteriormente, al emplear la prueba no paramétrica $U$ de Mann- Whitney, como se observa en la Tabla 3, con una 
significancia del .05 se puede afirmar que las familias nucleares tienen una mejor actitud hacia la salud $(.0025<.05)$, el temperamento $(.0245<.05)$ y el género $(.009<.05)$ del niño adoptable que las familias monoparentales. En la misma medida, con respecto a la etnia y la edad, si bien las actitudes de cada tipo de familia son diferentes, no son significativas estadísticamente. Teniendo en cuenta estos datos, se podría afirmar que las familias nucleares reaccionan con mayor favorabilidad hacia el hecho de que el niño adoptable presente o no algún tipo de discapacidad o enfermedad física o mental, al hecho de que el menor sea introvertido o extrovertido y respecto a que sea mujer u hombre.
En la misma medida, con una significancia del .05 se puede afirmar que las familias nucleares tienen una mejor actitud a nivel cognitivo $(.0015<.05)$ y conductual $(.025<.05)$ hacia las características del niño adoptable en comparación con las familias monoparentales. En vista de los datos, se puede afirmar que las familias nucleares tienen creencias, opiniones y conocimientos más favorables hacia las características del niño adoptable y están más dispuestos a adoptar a estos menores respecto a las monoparentales; destacando además que ambos tipos de familias no difieren de forma significativa en cuanto a los sentimientos a favor de las características del menor (Tabla 4).

Tabla 3

Estadísticos de contraste de tipos de familia hacia las características del niño adoptable

\begin{tabular}{cccccc}
\hline & Etnia & Edad & Salud & Temperamento & Genero \\
\hline $\begin{array}{c}\text { U de Mann-Whitney } \\
\text { Sig. asintótica (Unilateral) }\end{array}$ & .319 & .3895 & .0025 & .0245 & .009 \\
\hline
\end{tabular}

Tabla 4

Estadísticos de contraste de componentes de actitud

\begin{tabular}{cccc}
\hline & Actitud afectivo & Actitud cognitivo & Actitud Conductual \\
\hline $\begin{array}{c}\text { U de Mann-Whitney } \\
\text { Sig. asintótica (unilateral) }\end{array}$ & .0795 & .0015 & .025 \\
\hline
\end{tabular}

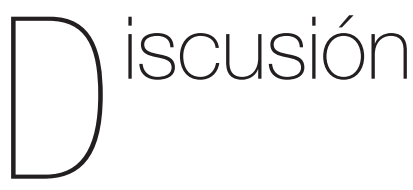

Teniendo en cuenta que el objetivo general de este estudio fue describir las actitudes hacia los niños adoptables por familias monoparentales y nucleares de estratos altos en la ciudad de Bogotá, además de comparar las actitudes de estos tipos de familias siendo coherentes con el tipo de estudio, se halló que las familias nucleares tuvieron mejores actitudes a nivel cognitivo y conductual, así como mejores actitudes hacia la salud, el temperamento y el género del niño adoptable.

De acuerdo a los hallazgos de esta investigación, y considerando la 
literatura anterior, como las investigaciones de Dave Thomas Foundation (2007) y Forward Scout (2015), desarrolladas en Norteamérica y Australia, así como los resultados de los estudios realizados en Bogotá, como los de Aguirre et al. (2007), Castañeda (2004) y Riveros (2008), no se aprecia concordancia, pues en esta investigación se evidenció un mayor predominio de actitud neutra en el componente, tanto afectivo como cognitivo, y no una actitud favorable y opiniones positivas como lo encontraron estos autores.

Ahora, si bien se evidenció que en la gran mayoría de familias predomina una actitud neutra a nivel cognitivo, también se halló que hubo familias que, aunque en menor medida, tomaron una actitud negativa en este componente, es decir, estas familias manifestaron conocimiento y creencias en contra de las características del niño, así como en los hallazgos de Dave Thomas Foundation (2007), pues es posible que estas familias tengan temor al percibir los problemas de salud, emocionales y comportamentales de los niños y sentirse incapaces de pagar por su cuidado y educación, al mismo tiempo que se concuerda con los hallazgos de Dave Thomas Foundation (2013), ya que muy seguramente las personas que reaccionan negativamente frente a las características del menor podrían pensar que los niños en condición de adoptabilidad tienden a tener problemas de vinculación, comportamiento y autocontrol.

Al evidenciar que la mayor parte de las familias nucleares y monoparentales presentaron, a nivel conductual, una actitud neutra (no se inclinan a adoptar a estos menores, ni tampoco a dejarlos en abandono), no se aprecia correspondencia con los hallazgos de Forward Scout
(2015), puesto que una parte significativa de familias presentaban una actitud negativa en este componente, lo que se aprecia al encontrar que dichas familias no consideraban adoptar.

Por otro lado, en relación al estudio de Aguirre et al. (2007), se observan resultados similares, pues, al igual que estos autores, se aprecia una actitud neutra en su componente conductual o un nivel medio de disposición a adoptar a estos menores. Así, existe la posibilidad de que esta actitud se deba a que muchas de las personas piensan en la adopción como una mera satisfacción de las necesidades propias, es decir, es posible que haya más disposición a adoptar en personas infértiles; no obstante, en ausencia de dicha condición, podría ser menos probable que una familia se motive a adoptar pensando exclusivamente en procurar el bienestar y la felicidad de los menores.

Por otro lado, al no hallar concordancia con los resultados de investigaciones llevadas a cabo en Norteamérica y Australia, uno de los factores que pueden dar razón de tales divergencias es el contexto, puesto que la cultura y la dinámica social de Bogotá pueden diferir de la de tales lugares. En consecuencia, como la cultura ejerce influencia significativa en los conocimientos y percepciones de los individuos en una sociedad, ésta puede llegar a intervenir de cierta forma en la adquisición de dichas actitudes (Baron, 2005).

Por lo demás, al observar diferencias con estudios llevados a cabo en el contexto bogotano, cuyos participantes fueron en esencia padres pre adoptantes y adoptivos, dichas particularidades de las familias participantes pueden ejercer 
cierta influencia, ya que es posible que las actitudes de quienes están actualmente adoptando o realizando la solicitud de adopción, sean distintas a las de personas que no han tenido alguna relación con este proceso, dado que, al tomar la decisión de adoptar, lleva consigo una favorabilidad. De acuerdo a ello, se sugiere hacer más estudios en Bogotá con familias no implicadas dentro de la adopción.

Ahora bien, en cuanto a las actitudes hacia cada característica del niño adoptable, la mayor parte de las familias, tanto nucleares como monoparentales, mostraron una actitud negativa frente al hecho de que el niño fuese menor de 5 o mayor de 8 años, coincidiendo esto con los resultados de Dave Thomas Foundation (2013) en el sentido de mayores probabilidades de adopción de niños menores de 3 años, teniendo una percepción negativa de adoptabilidad de un niño mayor de 12 años.

De igual modo, el hallazgo relacionado con que la mayor parte de familias asumieron una actitud positiva hacia la etnia del niño adoptable, concuerda con los resultados en las investigaciones de la Dave Thomas Foundation (2013) y Castañeda (2004), en donde la percepción de la raza fue considerada como un aspecto secundario ante la decisión de adoptar. En contraposición, no es consistente con el trabajo de Riveros (2008), en donde se evidenció una actitud neutra respecto a la etnia.

Frente al género, se presentaron discrepancias con la investigación de Dave Thomas Foundation (2013), al evidenciar que no predominó una actitud negativa en las familias de este estudio hacia el hecho de que el menor fuese niño o niña, contrario a lo encontrado por esta fundación donde se destacó una preferencia por las niñas y una percepción negativa hacia los niños. Por otro lado, se concuerda con los hallazgos de Riveros (2008), pues se evidenció una mayor proporción de familias tanto con una actitud neutral como con una positiva.

Teniendo en cuenta la actitud adoptada por familias nucleares y monoparentales respecto a la salud de los menores (la cual oscila entre neutra y negativa), dichos hallazgos podrían soportar los argumentos de Aguirre et al. (2007), al considerar tales autores que la falta de disposición a adoptar podría deberse al temor del origen genético, historia de vida y salud, en particular a esta última, ya que las personas se sentían incapaces de afrontar y suplir los gastos económicos requeridos en el caso de que los niños adoptables tengan deficiencias en su salud, por la cual se refleja una percepción negativa frente a dicha característica. Adicionalmente, se encuentra correspondencia con Riveros (2008), que también indica una actitud neutra frente a esta característica.

En cuanto al temperamento, en ambos tipos de familias predomina una actitud positiva hacia éste, así como en el trabajo de Riveros (2008), mientras que no se evidencian los mismos resultados en investigaciones como las de Dave Thomas Foundation (2013) y Aguirre et al. (2007), quienes observaron que la mayoría de los participantes consideran a los niños adoptables como difíciles porque provienen de circunstancias complicadas, tales como la delincuencia, y al cuestionarse sobre su historia de vida y su efecto en la conducta, los menores tendrán mayor posibilidad de presentar problemas de autocontrol o vinculación. 
Los datos de este estudio sobre las diferencias significativas en cuanto a las actitudes de familias monoparentales y nucleares hacia las características del niño adoptable, coinciden con el estudio de Riveros (2008) y Aguirre et al. (2007) donde se evidenció la existencia de diferencias significativas en el perfil de actitudes de personas casadas, solteras, viudas y divorciadas; sin embargo, Riveros (2008) también halló diferencias en los dos tipos de familias hacia la etnia del niño.

En la misma medida tampoco se corresponde en su totalidad con los hallazgos de Aguirre et al. (2007), pues si bien en este estudio se hallaron diferencias significativas entre ambas familias a nivel conductual, éstas indicaron que las familias nucleares tienen mayor disposición a adoptar que las familias monoparentales, mas no al contrario como lo evidenciaron estos autores.

Teniendo en cuenta las estadísticas del Instituto Colombiano de Bienestar Familiar (2015), este estudio refleja cómo las actitudes son coherentes con éstas, pues se evidencia cómo las familias no presentan en mayor o menor medida una disposición a adoptar a estos menores, por lo que sus comportamientos prosociales no están bien definidos (aspecto que refleja el componente conductual de las actitudes). En la misma medida, se aprecia que esta falta de hacer actividades que benefician a estos menores puede estar relacionada con los esquemas y prejuicios que movilizan a las personas, en este caso, la presencia de prejuicios frente al hecho de que estos menores formen parte de un grupo social en desamparo y que presenten ciertas características.
Al encontrar que las familias monoparentales tienen más actitudes negativas frente a cada una de las características del menor adoptable, se puede afirmar que éstas tienen mayores prejuicios, lo que podría ser explicado a partir de los esquemas de conocimientos y creencias que tengan, por lo que también se explica su menor disposición a adoptar en comparación con las familias nucleares. De esta forma, sus esquemas podrían tener efectos en el pensamiento social acerca de los niños adoptables siendo moldeados por la cultura, que no siempre son favorables dependiendo el punto de vista que éstas tengan (Baron, 2005).

Las familias monoparentales presentaron actitudes más negativas que las familias nucleares, siendo en esencia significativas hacia la salud, temperamento y género; así, se podría apreciar, teniendo en cuenta lo afirmado por Rosser y Moya (2001), que, al ser estas familias conformadas por un solo progenitor y sus hijos, perciban al niño adoptable de forma diferente a las otras familias; así mismo, en el caso de que tales menores tengan alguna discapacidad o enfermedad puede llevar a que, según su condición económica y demás circunstancias ya comentadas, reaccionen con cierta negatividad hacia tales características.

Un ejemplo de los esquemas que pueden tener las familias monoparentales, aunque éstas sean de estratos altos, es el hecho de que, al saber que tienen que distribuir su tiempo para sus actividades laborales y para las actividades de su casa y sus hijos sin ayuda de su pareja, pueden llegar a considerar de forma negativa la adopción de un niño con discapacidades, pues, como mencionan Cordero et al. (2013), podría haber incertidumbre del 
padre o madre de no poder sentirse capaz de suplir la totalidad de las necesidades de su nuevo hijo, lo que no ocurriría en el caso de una familia nuclear; a su vez, el hecho de que socialmente se perciba a estos niños como anormales puede llevar a que se tome una posición prejuiciosa frente a ciertas características de los menores y, en consecuenci,a no se sientan motivadas a adoptar, resaltando que se trataría de un comportamiento prosocial que beneficia a este grupo (Baron, 2005).

Bajo estas circunstancias, es posible que dentro de la cultura colombiana exista exclusión social relacionada con el prejuicio, razón por la cual precisamente aquellos menores cuyas características no son tan deseadas socialmente se queden esperando por largos periodos de tiempo por un hogar donde encontrar amor y protección, cultura que a la vez influye en la conformación de pensamientos tales como "son niños difíciles" (como se ha encontrado en la literatura), muy arraigados en la idea de que estos menores podrían llegar a ser delincuentes. Vale la pena resaltar que este tipo de pensar lleva a que en muchas ocasiones, si bien se perciba con lastima a estos menores, no hay una disposición a adoptarlos puesto que la persona no se siente capaz de involucrase a sí misma en tal decisión ya que es posible que de trasfondo exista una negatividad hacia ellos viéndose reflejada una forma de prejuicio sutil, en la cual, aunque no se manifiesta abiertamente tal negativa, es probable observarla a la hora de llevar a cabo un comportamiento (Frías et al., 2009).

Antes de finalizar es importante resaltar que los procesos cognitivos que desarrolla el individuo tienen gran importancia en la creación de estas representaciones sociales, ya que permiten categorizar - clasificar la información percibida y los significados. Estos procesos se evidencian cuando se percibe a un menor adoptable y se piensa que es un niño en abandono y, en consecuencia, se clasifica como un niño delincuente o un niño difícil basándose en el hecho de que dichos niños se recogieron en las calles y es allí donde viven los delincuentes.

Finalmente, para posteriores investigaciones se recomienda tener muestras más amplias, además de llevar a cabo más investigaciones con familias que no estén implicadas en el proceso de adopción, es decir, que no hayan adoptado ni que sean pre adoptantes, todo ello con el objetivo de llegar a recoger mayor evidencia que soporte los resultados de esta investigación.

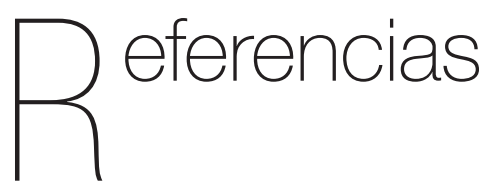

Aguirre, M., Hurtado, D., Márquez, E., \& Lombana, M. (2007). Factores que influyen en las familias bogotanas para no adoptar niños, niñas y recomendaciones al ICBF para transformarlos. Universidad de la Salle, Bogotá D.C.

Alonso, A. (1999). Formas, cambios y tendencias en la organización familiar en Colombia. Universidad Javeriana. Bogotá D.C.

Baron, R. (2005). Psicología social. (10ª Ed.). Madrid: Pearson Educación. 
Belén, A. (2005). Modelos y realidades de la familia actual. (10. Ed.). Bogotá: Fundamentos.

Bustamante, A. (2013). La adopción: aspectos psicológicos y educativos para el ciclo básico de la Facultad de Educación de la Universidad de Cantabria. Santander, España: Universidad de Cantabria.

Castañeda, S. (2004). Actitud de los padres adoptantes hacia el proceso de adopción. Universidad Rafael Urdaneta, Maracaibo.

Cordero, R., Yánez, A., \& Falconi, G. (2013). Estereotipos que amenazan la construcción de nuevas familias. La adopción otra forma de nacer. Universidad San Francisco de Quito.

Corte Constitucional (2011). Sentencia C-577/11. Recuperado de http:// www.corteconstitucional.gov.co/ relatoria/2011/C-577-11.htm

Dave Thomas Foundation. (2007). National Foster Care Adoption Attitudes Survey, Ohio.

Forward, S. (2015). Modern families: attitudes and perceptions of adoption in Australia. Australia: Adopt Change.

Frías, D., Monterde i Bort, H. \& Peris, F. (2009). La medida del prejuicio manifiesto y sutil. Interpsiquis, 1, 1-9. Recuperado de http://www.psiquiatria.com/articulos/ psicologia/39607

Harris Interactive (2013). National Foster Care, Adoption Attitudes Survey, Executive Summary \& Detailed Findings. U.S.A.: Dave Thomas Foundation for Adoption.
Harris Interactive (2007). National Foster Care, Adoption Attitudes Survey, Executive Summary. U.S.A.: Dave Thomas Foundation for Adoption.

Hernández, S., Fernandez, C., \& Baptista, L. (2010). Metodología de la investigación. México: McGraw-Hill.

Instituto Colombiano del Bienestar Familiar. (2015). Comunicado de prensa. Oficina asesora de comunicaciones.

Kassin, S., Fein, S. \& Markus, H. (2010). Psicología social. México: Cengage Learning Editores.

López, A. M., \& Garrido, D. F. (2009). Diagnósticos de las herramientas comunicativas del ICBF para promover la adopción de niños de difícil adopción para el ciclo básico de la Facultad de Comunicación y Lenguaje. Pontificia Universidad Javeriana, Bogotá.

Meneses, L., Arenas M., \& Pino S. (2005). Pobreza y abandono parental. Macropoyecto Sujetos y Diversidad: Una mirada desde el Desarrollo Humano. Recuperado de http://ridum.umanizales. edu.co:8080/jspui/bitstream/6789/954/4/ Pobreza\%20y\%20abandono\%20parental. pdf

Ministerio de la Protección Social. Ley 1090 de 2006. Recuperado de http://colpsic.org.co/ aym_image/files/LEY_1090_DE_2006.pdf

Miranda, J. (1999). La adopción como institución jurídica y medida de protección por excelencia para el ciclo básico de la Facultad de Ciencias Jurídicas y Derecho. Pontificia Universidad Javeriana, Bogotá. 
Myers, D. (2000). Psicología social. (6ª . Ed.). Bogotá: Mc Graw Hill- Interamericana.

Palacios, J. (2009). La aventura de adoptar. Guía para solicitantes de adopción internacional. Madrid, España: Ministerio de Sanidad y Política Social.

Pérez, M. (2016). Derecho de familia y sucesiones. Colección Cultura Jurídica. México: UNAM.

Pineda, F., \& Moreno, J. (2008). Factores psicosociales asociados al abandono infantil de un grupo de adolescentes institucionalizadas en un centro de protección en la ciudad de Bogotá. Psychologia: avances de la disciplina, (2), 151-182.

Riveros, F., Bohórquez, D., López, S., \& Sepúlveda, E. (2015). Diseño y validación de un instrumento para medir las actitudes frente a la labor profesional del psicólogo. Revista Iberoamericana de Psicología: ciencia y tecnología, 8(2), 55-66.
Riveros, N. (2008). Actitudes de las personas pre-adoptantes hacia las características del niño adoptable en la ciudad de Lima, Perú. (Tesis de pregrado). Recuperado de http://cybertesis.unmsm.edu.pe/ bitstream/handle/cybertesis/1361/ Riveros_an.pdf?sequence $=1$ \&isAllowed $=\mathrm{y}$

Robbins, S. (2004). Comportamiento organizacional. (10 Ed.) Pearson: México.

Rosser, L. \& Moya, M. (2001). Familias monoparentales e idoneidad para la adopción. Intervención Psicosocial, 10(2), 209-220.

UNICEF. (2006). Convención sobre los derechos de los niños. Recuperado de http://www.un.org/es/events/childrenday/ pdf/derechos.pdf 